\title{
Are Axonal Mitochondria Really Enriched at Nodes of Ranvier? A Three Dimensional Serial Ion Ablation SEM (FIB/SEM) Study.
}

Grahame Kidd*, Nobuhiko Ohno ${ }^{*}$, Amir Avishai ${ }^{* *}$, Nanthawan Avishai**, Arthur H. Heuer ${ }^{* *}$, Xinghua Yin*, and Bruce D Trapp*.

*Dept. of Neurosciences, Lerner Research Inst., Cleveland Clinic Fndn, Cleveland, Ohio 44120

**Dept. of Mat. Sci. and Eng., Case Western Reserve University, Cleveland, OH, 44106

To meet the energy demands of nerve conduction, small mitochondria are transported from the neuronal cell body and enriched at mitochondrial stationary sites located throughout the axon. Determining whether and how myelination locally regulates mitochondrial structure and function in axons is fundamental to understanding axonal biology and pathology [1]. Nodes of Ranvier are the sites of action potential propagation, and previous EM studies of myelinated axons have suggested that axons are enriched in nodal axonal cytoplasm (axoplasm). Paradoxically, several studies noted that mitochondria were frequently absent at nodes of Ranvier [2,3]. Numbers of nodes with and without mitochondria had not been quantified, presumably to difficulties in obtaining extensive 3D EM datasets including entire nodes.

New, highly automated approaches using focused ion beam system have provide three dimensional EM datasets by serial ion ablation SEM (SIA-SEM) [4], and have proven valuable for studying myelinated axons [5]. In this study we utilized SIA-SEM to investigate the contribution of mitochondria to nodal axoplasm in small myelinated axons from mouse optic nerve, rat cerebellar white matter and rat corpus callosum. Tissues were glutaraldehyde fixed by perfusion and post-fixed and en bloc stained using the method of Deerinck et al. [6]. Tissues were embedded in Durcupan resin and samples were imaged using either a Nova-200 Nanolab or Helios 650 Dual Beam FIB (FEI) systems using G2 or G3 "Slice\&View" operating software. Serial images of the block faces were generated by repeated cycles of milling (1nA gallium ion beam current) and imaging ( $2 \mathrm{kV}, 21-84 \mathrm{pA})$ using high-resolution/immersion lens (mode 2) in backscattered electron mode. Sets of 250-500 images at $40 \mathrm{~nm}$ steps ("slice thickness") were obtained at 6.5 or $17 \mathrm{~nm} /$ pixel resolution, producing fields of $\sim 13-35 \mu \mathrm{m}$ wide and $8-20 \mu \mathrm{m}$ deep. These imaging conditions maximized axon sample length while still discriminating between individual mitochondria. Images were registered, corrected for aspect ratio, and derivative stacks were generated using ImageJ/FIJI software, and mitochondria and axons were traced and analyzed using Reconstruct software.

Both presence/absence of mitochondria and their relative density in nodal axoplasm varied greatly between axons. In cerebellar white matter from postnatal day 30 (P30) rats, 39\% of nodal axoplasm contained no mitochondria, $40 \%$ had a single mitochondrion, and $21 \%$ had $2-5$ mitochondria ( $\mathrm{n}=33$ axons). In corpus callosum axons from adult rats, only $30 \%$ of nodes had one or more mitochondria present (9/30), and in P30 mouse optic nerve, only a third (7/21) had axonal mitochondria at the node. Axons in each tissue were $0.5-2 \mu \mathrm{m}$ in diameter, which is a typical size for myelinated axons in the CNS. Ratios of mitochondrial volume to axonal volume have been frequently used to compare the concentrations of mitochondria in different axonal regions. In P30 cerebellar nodal axoplasm, the percentage of axonal volume occupied by mitochondria $(\mathrm{mV} / \mathrm{aV})$ values varied extensively and were not normally distributed. This 
observation is not surprising considering that many nodes had no mitochondria while others had more than one. In addition, the reduced axonal volume at the node skewed the $\mathrm{mV} / \mathrm{aV}$ values for the nodes containing mitochondria. Because nodal $\mathrm{mV} / \mathrm{aV}$ values are not normally distributed, when averaged across multiple axons (as reported in some earlier studies), their mean values were very misleading: the average nodal $\mathrm{mV} / \mathrm{aV}(10.2 \%)$ was 1.3 -times the internodal average, whereas the median value (a more appropriate nonparametric measure of centrality) was 0.6times that of internodal regions.

Our data demonstrate quantitatively that many or most axons in these CNS fiber tracts have no mitochondria in nodal axoplasm. The results are broadly consistent with qualitative observations in the literature $[2,3,7,8]$. Although mitochondria are not enriched in nodal axoplasm, many nodes do contain mitochondria and their presence may depend upon multiple factors, including electrical activity and fiber tract location. As demonstrated by comparing cerebellum and optic nerve axons, the percentage of nodes containing mitochondria can vary in different CNS fiber tracts and may also differ between CNS and PNS axons [3]. For most CNS axons, however, mitochondria are neither concentrated at nodes nor appear essential components of nodal axoplasm.

\section{References}

[1] B. D. Trapp and P. K. Stys. Lancet Neurol. 8 (2009) 280.

[2] J. M. Edgar et al., J. Neurosci. Res. 86 (2008) 2250.

[3] G. A. Perkins and M. H. Ellisman, J Struct Biol 173 (2011) 117.

[4] G. Knott et al., J. Neurosci. (2008) 28, 2959.

[5] G. J. Kidd et al., Microscopy Today 18 (2010) 18.

[6] T. J. Deerinck et al., Microscopy and Microanalysis 16 (2010) 1138.

[7] C. Fabricius et al., J. Neurocytol. 22 (1993) 941.

[8] C. H. Berthold et al., J Neurocytol. 22 (1993) 925. 